\title{
O Gênero Erythroxylum P. Browne (Erythroxylaceae) do Estado do Paraná, Brasil
}

\author{
Joalice de Oliveira Mendonça ${ }^{1 *}$; Armando Carlos Cervi ${ }^{2}$ e Olavo Araújo Guimarães ${ }^{2}$ \\ ${ }^{1}$ Doutoranda do Curso de Pós-Graduação em Botânica, UNESP. Cx. Postal 510 - 18618-000, Botucatu, São \\ Paulo, Brasil; ${ }^{2}$ Prof. do Departamento de Botânica, Universidade Federal do Paraná. Cx. Postal 19031 - 81531 - \\ 970 - Curitiba, Paraná, Brasil.
}

\begin{abstract}
An inventory of the Erythroxylum's species from the State of Paraná, Brazil, was done and the following species were found: E. ambiguum Peyr., E. amplifolium (Mart.) O.E. Schulz, E. anguifugum Mart., E. argentinum O.E. Schulz, E. buxus Peyr., E. campestre A. St.-Hil., E. cuneifolium (Mart.) O.E. Schulz, E. cuspidifolium Mart., E. deciduum A. St.-Hil., E. gonocladum (Mart.) O.E. Schulz, E. nanum A. St.-Hil., E. pelleterianum A. St.-Hil., E. microphyllum A. St.-Hil., E. myrsinites Mart. and E. suberosum A. St.-Hil.. All of then are taxonomically revised and a new synonym is presented: E. cuneifolium (Mart.) O.E. Schulz var. squarrosum O.E. Schulz as synonym of E. cuneifolium (Mart.) O.E. Schulz. Is also discussed the validity of the genus name Erythroxylon Linnaeus against Erythroxylum P. Browne.
\end{abstract}

Key words: Erythroxylaceae, Erythroxylum, Flora, Paraná, Taxonomy

\section{INTRODUÇÃO}

As Eritroxiláceas com 4 gêneros e 200 espécies pantropicais, estão representadas nas Américas, onde ocorre o maior número de espécies, apenas pelo gênero Erythroxylum P. Browne. Os demais gêneros, Aneulophus Benth., Nectaropetalum Benth. e Pinacopodium Exell \& Mendonça, ocorrem no Continente Africano e totalizam cerca de 10 espécies (Cronquist, 1981). Certamente, Erythroxylum coca Lam. e suas variedades, sejam as plantas mais conhecidas dessa família, por seu cultivo para obtenção da cocaína.

Este levantamento, assim como o realizado por Mendonça (1996), teve como principais objetivos, a revisão taxonômica, a ampliação do conhecimento da distribuição geográfica e a apresentação de uma chave de identificação das espécies que ocorrem no Estado do Paraná.

$\mathrm{O}$ número atual de espécies do gênero Erythroxylum ocorrentes no Brasil situa-se em torno de 127, podendo alterar-se à medida que novos estudos forem desenvolvidos. Dessas, 15 ocorrem no Estado do Paraná.

\section{MATERIAL E MÉTODOS}

Podemos distinguir no Estado do Paraná, cinco grandes regiões geomorfológicas em um corte partindo, do Oceano Atlântico (leste) para o Oeste: 1- Litoral; 2- Serra do Mar; 3- Primeiro Planalto ou Planalto de Curitiba; 4- Segundo Planalto ou Planalto de Ponta Grossa e 5Terceiro Planalto ou Planalto de Guarapuava. Coletou-se material botânico e informações de campo em todas essas regiões.

O material coletado foi incorporado ao herbário do Departamento de Botânica da Universidade Federal do Paraná (UPCB).

Os trabalhos de coleta e herborização, foram realizados segundo as técnicas de Radford et al. (1974).

\footnotetext{
* Autor para correspondência
} 
Além do material coletado, foram examinados materiais de outros herbários, tais como: BOTU, EFC, FUEL, HAS, HBR, ICN, M, MBM, PACA, PKDC, RB , SP, UEC, UPCB. As siglas estão de acordo com HOLMGREN et al. (1990). Foram examinados também, tipos nomenclaturais e fotografias dos tipos.

A chave analítica foi elaborada baseando-se em caracteres morfológicos reconhecíveis, tanto no material fresco quanto no herborizado.

Do material examinado, foi citado apenas um exemplar de cada localidade do Estado do Paraná. E quando esse material era escasso, foi examinado material adicional de outros estados.

\section{RESULTADOS E COMENTÁRIOS}

As espécies do gênero Erythroxylum que ocorrem no Estado do Paraná, pertencem a 4 seções: Archerythroxylum O.E. Schulz, Macrocalyx O.E. Schulz, Microphyllum O.E. Schulz e Rhabdophyllum O.E. Schulz .

Chave para identificação das espécies do gênero Erythroxylum do Estado do Paraná:

1. Estípula enérvea ou com nervuras pouco visíveis

2. Estípula persistente, coriácea

3. Folha de 50-100mm comprimento, com pecíolo evidentemente maior que a estípula

4. Folha com ápice arredondado, emarginado, pecíolo longo (5-10mm) e delicado. E. argentinum

4: Ápice com outra forma, pecíolo mais curto $(2-7 \mathrm{~mm})$ crasso

5. Ápice cuspidado, estípula 1,2$2 \mathrm{~mm}$, nervuras de quarta ordem pouco visíveis. E. cuspidifolium

5: Ápice de agudo a obtuso, estípula de 2-2,5mm, nervuras de quarta ordem nítidas E. anguifugum

3: Folha de até $55 \mathrm{~mm}$ de comprimento, pecíolo menor ou pouco maior que a estípula

6. Folhas $10-55 \mathrm{~mm}$ comprimento $\mathrm{x} 5$ 27mm largura, nervuras secundárias conspícuas

7. Lenticelas arredondadas ou elípticas, ramos cilíndricos ou um pouco comprimidos.
8. Estípula 2-setulosa bem maior que o pecíolo, nervuras laxas, retículo das nervuras bem aberto...............E. cuneifolium

8: Estípula 3-setulosa, eqüilonga ou menor que o pecíolo, nervuras mais congestas, retículo das nervuras bastante denso

E. amplifolium

$\begin{array}{llr}\text { 7: } & \text { Lenticelas em fendas } \\ \text { longitudinais, } & \text { bem longas, ramos } \\ \text { angulosos........................................ gonocladum }\end{array}$

6: Folhas $5-13 \mathrm{~mm}$ comprimento x 2$4 \mathrm{~mm}$ largura, nervura secundária inconspícuas........................... E. microphyllum

2: Estípula decídua e
membranácea.................................E. ambiguum 1: Estípula nervada longitudinalmente

9. Córtex suberoso, lacínios do cálice largoovais. E. suberosum

9: Córtex não suberoso, lacínios triangulares 10. Ápice das estípulas novas nitidamente fimbriados. E. pelleterianum

10: Ápice das estípulas novas não ou levemente fimbriados

11. Folhas adultas membranáceas, pedicelo de 4-7mm E. myrsinites

11: Pedicelo de 8,4(15)-23mm

\section{E. buxus}

12. Folhas adultas de cartáceas a coriáceas

12: Estípula de eqüilonga a menor que o pecíolo E. deciduum

pecíolo

13. Estípula maior que o

13: Nervuras secundárias e terciárias pouco salientes ou impressas, pouco nítidas na face superior. E. campestre

14. Nervuras secundárias, terciárias e quaternárias bem salientes e nítidas na face superior. E. nanum

I - Seção - Archerythroxylum O.E. Schulz

1. Erythroxylum ambiguum Peyr. in Mart. Fl. Bras. XII. 1: 142. 1878; O.E. Schulz in Engler Das Pflanzenr. IV. 134. 29: 80. 1907; Amaral in Fl. Ilust. Catarinense Fasc. ERIT: 37. est. 8. 1980.

Nome vulgar: fruta-de-pomba.

Fenologia: Floresce de agosto a novembro e frutifica de setembro a dezembro. 
Distribuição: Santa Catarina, Paraná, São Paulo, Rio de Janeiro e Minas Gerais. Espécie componente da encosta atlântica, vertentes da face norte da Serra da Mantiqueira e capoeiras próximas da orla marítima.

Uso: madeira utilizada como lenha e sementes servem de alimento às aves (Corrêa, 1984).

Comentários: Esta espécie quando desenvolvese em locais mais sombreados, apresentam folhas grandes, com 120mm de compr., e textura membranácea. Quando vegeta em condições de receber maior radiação solar, nas capoeiras e orlas de matas, suas folhas são menores e cartáceas.

Material examinado: PARANÁ: Antonina, Bairro Alto, X.1989, O.S. Ribas 181 (MBM). Guaraqueçaba, Morro do Quitumbê, VIII.1995, J.O. Mendonça \& A. Uhlmann 34 (UPCB). Guaratuba, Colina Limeira, X.1971, G. Hatschbach 27553 (UEC). Morretes, Faz. do IAPAR, VII.1995, J.O. Mendonça et al. 27 (UPCB). Paranaguá, Alexandra, II.1968, G. Hatschbach 18616 (MBM). Porto de Cima, XI.1908, P. Dusén 7040 (M).

2. Erythroxylum amplifolium (Mart.) O.E. Schulz in Engler Das Pflanzenr. IV. 134. 29: 107. 1907; Amaral in Fl. Ilust. Catarinense Fasc. ERIT: 23. est. 4 e 5. 1980.

Nome vulgar: fruta-de-pomba.

Fenologia: Floresce de agosto a outubro, frutifica de setembro a dezembro. É muito comum a ocorrência de botões, flores e frutos na mesma planta.

Distribuição: Rio Grande do Sul, Santa Catarina, Paraná, São Paulo, Rio de Janeiro, Minas Gerais, Distrito Federal, Maranhão e segundo O.E. Schulz (1907), Uruguai. No Estado do Paraná, habita preferencialmente a zona litorânea, principalmente a restinga. Segundo Amaral Júnior (1980) como elemento raro, também ocorre na floresta do Alto Uruguai.

Uso: Apesar de Corrêa (1984), citar que a madeira desta espécie é utilizada para cabos de ferramentas, construção civil e marcenaria, tal uso não é possível, pois a espessura do caule não permite.

Material examinado: PARANÁ: Guaraqueçaba, Ilha das Peças, V.1994, J.O. Mendonça R.C. Tardivo 14 (UPCB). Paranaguá, Ilha do Mel, Praia Brasília, X.1993, J.O. Mendonça et al. 01 (UPCB). Pontal do Sul, Balneário Atami, XI.1989, S.R. Ziller 107 (EFC).

3. Erythroxylum anguifugum Mart. in Abhandl. Akad. München III. 2: 361. t. 5. 1843; Peyr. in Fl. Bras. XII. 1: 148. 1878; O.E. Schulz in Engler Das Pflanzenr. IV. 134. 29: 109. 1907.

Nome vulgar: fruta-de-pomba.

Fenologia: Floresce de agosto a abril, frutifica de setembro a abril. É freqüente encontrarmos plantas com flores e frutos ao mesmo tempo.

Distribuição: Paraná, São Paulo, Minas Gerais, Goiás, Mato Grosso do Sul, Mato Grosso, Pará, Paraguai, Bolívia e segundo Plowman (1984), Peru. No Estado do Paraná (nov. loc.), esta espécie somente foi coletada nas margens do Rio Paraná, onde predomina a floresta estacional semidecidual.

Usos: Fornece matéria tintorial preta; a fumaça da madeira, diz-se afugenta as cobras, e sua raiz é citada como útil contra a picada das mesmas (Corrêa, 1984).

Material examinado: PARANÁ: Guaíra, Sete Quedas, XI.1963, E. Pereira 7887 \& G. Hatschbach 10501 (MBM, RB). Icaraíma, Rio Paraná, Paredão das Araras, I.1967, G. Hatschbach 15783 (MBM). Porto Rico, Ilha Mutum, Rio Paraná, X.1994, D.C. Souza \& J. Cislinski 4 (UPCB).

4. Erythroxylum argentinum O.E. Schulz in Engler Das Pflanzenr. IV. 134. 29: 99. 1907; Amaral in Fl. Ilust. Catarinense - ERIT: 7. 1980; Sobral in Pesquisas-Botânica 38:11. fig. 1. 1987. 
Nome vulgar: cocão, concon, fruta-de-pomba e baga-de-pomba.

Fenologia: Floresce de setembro a dezembro, frutifica de outubro a janeiro.

Distribuição: Rio Grande do Sul, Santa Catarina, Paraná, São Paulo e Argentina. Segundo Occhioni \& Hatschbach (1972), ocorre nos ervais do primeiro planalto e floresta estacional semidecidual do segundo planalto do Estado do Paraná, incluso nos contrafortes da Serra do Mar. De acordo com Amaral Júnior (1980), no Estado de Santa Catarina, ocorre na floresta ombrófila densa e zona litorânea.

Usos: Embora, Corrêa (1984), cite que a madeira desta espécie é utilizada na construção civil e tornearia, tal uso não é possível, pois a própria espessura do caule não permite.

Material examinado: PARANÁ: Morretes, .V.1961, O. Almeida 55 (ICN). Joaquim

Távora, XI.1976, G. Hatschbach 39285 (MBM).

Material adicional examinado: SANTA CATARINA: Itapema, .XI.1996, A.C. Cervi 6202 (BOTU, UPCB). SÃO PAULO: Botucatu, .III.1997, A. Amaral Jr. et al. 211397 (BOTU). Sorocaba, .X.1996, J.O. Mendonça \& A. Amaral Jr. 43 (BOTU).

Comentários: O.E. Schulz (1907), apresenta 2 variedades para esta espécie: E. argentinum var. argentinum e E. argentinum var. calophyllum, com base no comprimento dos pecíolos e comprimento e largura das folhas. SOBRAL (1987), propõe a sinonimização de $E$. argentinum var. calophyllum baseando-se no fato de que as únicas diferenças dadas por O.E. Schulz, são as medidas das folhas e pecíolos e que essas diferenças podem estar relacionadas com fatores ambientais.

Observamos esta espécie no seu ambiente natural e, constatamos também essas variações como sendo decorrentes dos fatores ambientais, desta forma concordamos com a decisão tomada por Sobral (1987).

II - Seção Rhabdophyllum O. E. Schulz
5. Erythroxylum buxus Peyr., Fl. Brasil. 12(1): 137 t. 25, fig. 2. 1878.

Distribuição: Paraná (nov. loc.), São Paulo, Minas Gerais. Os representantes do litoral (coletados no Estado de São Paulo), apresentam folha, pecíolo e pedicelo bem maiores que os do interior do estado.

Material examinado: PARANÁ: Guaíra, P. Nac. Sete Quedas, IX.1980, J. Fontella Pereira 1156 (BOTU).

Material adicional examinado: SÃO PAULO: Eldorado Paulista, II.1995, H.F. Leitão Filho 32690 (SPF, UEC). Guareí, X.1996, A. Amaral Jr. \& J.O. Mendonça 42 (BOTU).

6. Erythroxylum campestre A. St.-Hil. in Fl. Bras. Merid. II: 97. 1829; Mart. in Abhandl. Akad. München III. 2: 373. t. 7. 1843; Peyr. in Fl. Bras. XII. 1: 173.1878 pro parte.

Nome vulgar: cabelo-de-negro, fruta-de-tucano.

Fenologia: Floresce de julho a novembro, frutifica de agosto a dezembro.

Distribuição: Paraná, São Paulo, Rio de Janeiro, Minas Gerais, Bahia, Mato Grosso do Sul, Mato Grosso, Goiás e Distrito Federal. No Estado do Paraná, somente foi encontrada em áreas de cerrado. Mais propriamente em campo cerrado.

Usos: Apesar de Corrêa (1984), citar que a madeira desta espécie é utilizada para obras de marcenaria, construção civil e carpintaria, tal uso não é possível, pois a espessura do caule não permite. Raiz e casca passam por purgativas.

Material examinado: PARANÁ: Arapoti, Chapadão Santo Antônio, X.1968, G. Hatschbach 19956 (MBM). Cianorte, Faz. Lagoa, G. Hatschbach 14245 (MBM). Jaguariaíva, P. Est. do Cerrado, XII.1994, J.O. Mendonça \& A. Uhlmann 29 (UPCB). São Jerônimo da Serra, XI.1970, G. Hatschbach 24813 (MBM). Sengés, Área de Preservação Permanente, III.1995, J.O. Mendonça A. Uhlmann 32 (UPCB). 
7. Erythroxylum deciduum A. St.-Hil. in Plant. Usuell. Bras. t. 69. fig. b. 1824; Fl. Bras. Merid. II: 95. 1829; O.E. Schulz in Engler Das Pflanzenr. IV. 134. 29: 57. 1907; Macbride in Fl. Peru, XIII. part. 3(2): 638. 1949; Machado in Raymondiana V: 50. tab. VIII. 1972; Amaral in Fl. Ilust. Catarinense Fasc. ERIT: 50. est. 12. 1980; Sobral in Pesquisas-Botânica. 38:17. fig. 1. 1987.

Nome vulgar: fruta-de-pomba, cocão, concon, baga-de-pomba.

Fenologia: Floresce de agosto a janeiro, frutifica de setembro a fevereiro.

Distribuição: Rio Grande do Sul, Santa Catarina, Paraná, São Paulo, Rio de Janeiro, Minas Gerais, Bahia, Mato Grosso do Sul, Mato Grosso, Goiás, Distrito Federal, Piauí, Peru, Paraguai e Argentina. Segundo Amaral Júnior (1980), a distribuição dessa espécie é bastante ampla. Tem seu limite meridional nas regiões de baixa altitude perto de Porto Alegre (RS) e na província de Corrientes, na Argentina. Desenvolve-se com freqüência no planalto até altitudes de 1000-1300 m. No Estado do Paraná, habita matas de galeria, capões e capoeiras e próximo a cerradões.

Usos: Embora Corrêa (1984), cite que a madeira desta espécie é utilizada na construção civil, marcenaria e carpintaria, isto não pode ser possível, pois a própria espessura do caule não permite.

Material examinado: PARANÁ: Arapoti, I.1965, L. B. Smith \& R. Klein 14642 (HBR). Campina Grande do Sul, Rio Divisa, X.1959, G. Hatschbach 6354 (HBR, PKDC). Campo Largo, Bateias, I.1978, G.Hatschbach 40757 (UPCB, MBM). Cantagalo, XI.1966, J.C. Lindman et al. 2816 (RB). Cascavel, P. Ecol. Paulo Gorski, XI.1988, J. da Cruz 176 (FUEL). Castro, Rio Cunhoporanga, XI.1988, S.M. Silva et al. 1655 (PKDC). Clevelândia, X.1969, G. Hatschbach 22711 (MBM). Colombo, IX.1986, A. Pegorano 78 (EFC). Curitiba, Centro Politécnico, XI.1993, J.O. Mendonça et al. 03 (UPCB). Guarapuava, Águas Santa Clara, X.1986, G. Hatschbach 50581 (MBM). Irati,
Colégio Est. Florestal, X.1972, P. Carvalho 64 (MBM). Jaguariaíva, Rio das Mortes, XI.1993, J.O. Mendonça 05 \& A.C. Cervi. (UPCB). Palmeira, Córrego da Anta, IX.1982, G. Hatschbach 45490 (MBM). Piên, XI.1990, C.B. Poliquesi et al. 22 (UEC). Piraí do Sul, Jararaca, IX.1975, G. Hatschbach 37067 (UEC, MBM). Piraquara, Floresta de Galeria do Rio Iraí, I.1993, S.R. Ziller \& A. Vicentin 384 (EFC). Ponta Grossa, P. Est. de Vila Velha, XI.1995, A.C. Cervi et al. 6023 (UPCB).Tibagi, Canyon Guartelá, X.1994, J.O. Mendonça \& A.C. Cervi 26 (UPCB). Tijucas do Sul, Saltinho, X.1971, G. Hatschbach 27583 (UPCB, MBM). Rio Negro, Lagoa Verde, X.1953, G. Hatschbach 3258 (HBR). São Mateus do Sul, VIII.1995, J.O. Mendonça 25 (UPCB). Sengés, Poço do Encanto, III.1995, J.O. Mendonça \& A. Uhlmann 24 (UPCB).

Comentários: Esta espécie, em estado fértil (no início da floração), encontra-se áfila. Quando começam a aparecer as primeiras folhas, apresentam-se membranáceas, somente na frutificação ou no estado vegetativo, é que vão se apresentar de cartáceas a subcoriáceas.

8. Erythroxylum myrsinites Mart. in Abhandl. Akad. München III. 2: 345. t. 4. 1843; Peyr. in Mart., Fl. Bras. XII. 1: 137. 1878; O.E. Schulz in Engler Das Pflanzenr. IV. 134. 29: 42. 1907; Amaral in Fl. Ilust. Catarinense Fasc. ERIT: 46. est. 11. 1980; Sobral in Pesquisas-Botânica. 38: 21. fig. 3. 1987.

Nome vulgar: fruta-de-pombo, cocão, concon, baga-de-pomba, fruta-de-pomba e na Argentina é conhecida como coca-de-índio.

Fenologia: Floresce de agosto até abril, frutifica de setembro a maio.

Distribuição: Rio Grande do Sul, Santa Catarina, Paraná, São Paulo, Minas Gerais, Argentina, Paraguai e Uruguai. No Estado do Paraná ocorre principalmente nas margens de rios, quase sempre na orla de matas, capões e capoeiras.

Usos: Casca adstringente, útil para curtume (Corrêa, 1984). 
Material examinado: PARANÁ: Balsa Nova, Rio Iguaçú, I.1985, P. Berry, T. Plowman \& F. Juarez 4490 (MBM). Curitiba, Centro Politécnico, XI.1993, J.O. Mendonça 02 (UPCB). Foz do Iguaçú, P. Nac. do Iguaçú, Macuco, X.1986, G. Hatschbach et al. 50642 (UPCB). Ivaí, Rio Palmital, X.1969, G. Hatschbach 22365 (MBM). Palmeira, Rio dos Papagaios, XI.1986, G. Hatschbach et al. 50826 (MBM). Piên, Poço Frio, X.1987, G. Hatschbach 51482 \& J.M. Silva (MBM). Pinhais, Autódromo, XII.1973, G. Hatschbach 33596 (MBM). Piraquara, Rio Iraí, XII.1992, S. R. Ziller 323 (MBM, EFC). Pitanga, X.1991, G. Hatschbach et al. 55745 (MBM). Rio Branco do Sul, X.1975, G. Hatschbach 37299 (MBM). Salto Osório, Rio Iguaçú, XII.1968, G. Hatschbach 20536 (MBM). Santa Helena, Rio São Francisco, XII.1977, G. Hatschbach 40527 (MBM). São Jerônimo da Serra, IX.1970, G. Hatschbach 24833 (MBM). União da Vitória, X.1966, G. Hatschbach 14904 (UEC, MBM).

9. Erythroxylum nanum A. St.-Hil. in Fl. Bras. Merid. II: 97. 1829.

Fenologia: Floresce de março a setembro, frutifica de outubro a janeiro.

Distribuição: Rio Grande do Sul, Paraná, São Paulo, Rio de Janeiro, Minas Gerais, Goiás e Distrito Federal. Ocorre nas áreas de cerrado, principalmente nas margens, e em estradas que os atravessam, resiste bem ao fogo e a danos mecânicos devido ao seu sistema subterrâneo.

Material examinado: PARANÁ: Jaguariaíva, Rio das Mortes, I.1965, L. B. Smith \& R. Klein 14775 (HBR). Sengés, Res. Ecol. da Pisa Florestal, J.O. Mendonça \& A. Uhlmann 28 III.1995 (UPCB).

10. Erythroxylum pelleterianum A. St.-Hil. in Fl. Bras. Merid. II: 100. t. 102. 1829; Mart. in Abhandl. Akad. München III. 2: 350. 1843; Peyr. in Martius, Fl. Bras. XII. 1: 139. 1878; O.E. Schulz in Engler Das Pflanzenr. IV. 134. 29: 39. 1907.

Nome vulgar: fruta-de-pomba, cocão.

Fenologia: Floresce de agosto a novembro.
Distribuição: Rio Grande do Sul, Paraná, São Paulo, Rio de Janeiro, Minas Gerais, Bahia, Mato Grosso do Sul, Goiás, Uruguai, Argentina e Paraguai. Segundo Amaral Júnior (1973), ocorre com mais freqüência nos cerrados e cerradões embora, ocorra também, em floresta estacional semidecidual, matas de galeria e capoeiras. É muito comum a queda das folhas no início da floração. No Estado do Paraná, foi encontrado apenas um registro de coleta, na região do primeiro planalto.

Usos: Suas folhas são estomáquicas (CORRÊA, 1984).

Material examinado: PARANÁ: Cerro Azul, Garaipós, I.1980, G. Hatschbach 42737 (MBM, UEC).

Comentários: Espécie facilmente reconhecida pela ramenta característica, disposta dísticamente nos ramos. Embora, não se tenha registros de coletas no Estado de Santa Catarina, é possível que ocorra nesse estado, pois ocorre nos Estados do Rio Grande do Sul e Paraná que fazem fronteira com o mesmo.

III - Seção Microphyllum O.E. Schulz

11. Erythroxylum cuneifolium (Mart.) O.E. Schulz in Engler Das Pflanzenr. IV. 134. 29: 121. fig. 21. 1907; Amaral in Fl. Ilust. Catarinense Fasc. ERIT: 18. est. 3. 1980; Sobral in Pesquisas-Botânica 38: 13. fig. 8. 1987.

E. cuneifolium (Mart.) O.E. Schulz var. squarrosum O.E. Schulz in Engler Das Pflanzenr. IV. 134. 29: 122. fig. 21. 1907. syn. nov.

Nome vulgar: cocão, concon, baga-de-pomba. fruta-de-pomba; coca-del-monte na Argentina.

Fenologia: Floresce de agosto a fevereiro, frutifica de setembro a março.

Distribuição: Rio Grande do Sul, Santa Catarina, Paraná, São Paulo, Rio de Janeiro, Minas Gerais, Mato Grosso do Sul, Mato Grosso, Goiás, Paraguai, Argentina e Bolívia. Habita desde formações florestais, como área de transição entre cerrado e floresta estacional semidecidual, e apresenta-se sempre com porte e 
folhas menores (folhas de cartáceas a coriáceas), que os indivíduos que ocorrem nas orlas de matas ciliares, que apresentam porte e folhas maiores e membranáceas. Ocorre também, na mata semidecidual do planalto, capões e capoeiras, sempre nas margens das mesmas e é espécie rupícola, de regiões rochosas e suas folhas apresentam-se de arredondadas a suborbiculares, córtex bastante modificado, grosso e um pouco suberificado. Os indivíduos que ocorrem no campo, próximo à mata ciliar, porém onde aparecem outras espécies de cerrado, totalmente expostos ao sol, apresentam folhas miúdas (semelhantes às folhas de $E$. microphyllum) e bastante coriáceas.

Usos: Apesar de CORRÊA (1984), citar que a madeira desta espécie é utilizada na carpintaria, marcenaria e obras de construção civil, isto não pode ser possível, pois a espessura do caule não permite.

Material examinado: PARANÁ: Arapoti, Rio das Cinzas, XI.1960, G. Hatschbach 6904 (MBM). Campo Mourão, X.1966, J.C. Lindman \& J. H. Haas 2739 (RB). Guaíra, Sete Quedas, III.1982, F. de Melo 346 (SP). Guarapuava, X.1994, R.M. Britez et al. 2118 (UPCB). Jaguariaíva, Rio das Mortes, XI.1993, J.O. Mendonça \& A.C. Cervi 06 (UPCB). Jardinópolis, P. Nac. Iguaçú, XI.1966, J.C. Lindman \& J.H. Haas 3417 (HBR). Palmas, XII.1980, G. Hatschbach 43496 (MBM). Ponta Grossa, Barra do Rio Pintangui, X.1959, G. Hatschbach 6344 (HBR, MBM). Tibagi, Canyon do Guartelá, XII.1993, J.O. Mendonça \& A.C. Cervi 04 (UPCB).

Comentários: E. cuneifolium tem grande variabilidade em seus aspectos morfológicos, variando de acordo com o ambiente ou formação vegetal em que se desenvolve. A observação desta variabilidade em $E$. cuneifolium (Mart.) O.E. Schulz var. squarrosum O. E. Schulz nos levou a desconsiderar esta variedade, sinonimizando-a.

12. Erythroxylum cuspidifolium Mart. in Abhandl. Akad. München III. 2: 359. t. 2. fig. 21. 1843; Peyr. in Fl. Bras. XII. 1: 152. 1878; O.E. Schulz in Engler Das Pflanzenr. IV. 134.
29: 116. 1907; Amaral in Fl. Ilust. Catarinense Fasc. ERIT: 13. est. 2. 1980; Sobral in Pesquisas-Botânica 38:16. fig. 5. 1987.

Nome vulgar: fruta-de-pomba, cocão, baga-depomba.

Fenologia: Floresce de agosto a dezembro, frutifica de outubro a março.

Distribuição: Rio Grande do Sul, Santa Catarina, Paraná, São Paulo, Rio de Janeiro, Espírito Santo, Minas Gerais e Bahia. Espécie característica da região litorânea, principalmente da encosta da floresta atlântica.

Usos: Apesar da literatura citar que a madeira desta espécie é utilizada na construção civil e carpintaria, isto não pode ser possível, pois a espessura do caule não permite.

Material examinado: PARANÁ: Antonina, Reserva Biol. de Sapitanduva, XI.1986, A.C. Cervi et al. 2399 (UPCB). Guaraqueçaba, Serrinha, I.1968, G. Hatschbach 18490 (MBM). Paranaguá, Ilha do Mel, Morro do Meio, X.1985, R. Britez 135 (FUEL).

13. Erythroxylum gonocladum (Mart.) O.E. Schulz in Engler Das Pflanzenr. IV. 134. 29: 122. 1907; Amaral in Fl. Ilust. Catarinense ERIT: 33. est. 7. 1980.

Nome vulgar: cocão, concon, baga-de-pomba, fruta-de-pomba.

Fenologia: Floresce de agosto a março, frutifica de setembro a abril.

Distribuição: Santa Catarina, Paraná, São Paulo, Rio de Janeiro, Minas Gerais. Habita desde a região litorânea nas margens dos rios, até o primeiro planalto paranaense.

Material examinado: PARANÁ: Campina Grande do Sul, Serra Virgem Maria, XI.1968, G. Hatschbach 20288 (MBM). Morretes, Rio dos Padres, XII.1982, P.I. Oliveira 714 (MBM). Quatro Barras, Rio Taquarí, IX.1967, G. Hatschbach 17150 (MBM). São José dos Pinhais, Colônia Santos Andrade, XII.1986, J. 
Cordeiro et al. 384 (UPCB).

Comentários: Segundo Amaral Júnior (1980), é espécie encontrada com maior freqüência nas regiões de campos rupestres e matas de altitude de Minas Gerais. Habita também a floresta tropical atlântica e encostas das montanhas. É bastante confundida com E. cuneifolium (de folhas miúdas), devido ao tamanho e forma das folhas, entretanto, as estípulas 3-setulosas, córtex rimuloso e ramos angulosos a distinguem facilmente da outra.

14. Erythroxylum microphyllum A. St.-Hil. in Fl. Bras. Merid. II: 100. t. 103. 1829; Peyr. in Mart., Fl. Bras. XII. 1: 133. 1878; O.E. Schulz in Engler Das Pflanzenr. IV. 134. 29: 120. 1907; Amaral in Fl. Ilust. Catarinense Fasc. ERIT: 28. est. 6. 1980; Sobral in Pesquisas-Botânica. 38: 19. fig.

E. perpusillum Fern. Casas, Pabstia, v.9, n.1, (Amaral Júnior \& Mendonça, 1998) syn. nov.

Nome vulgar: fruta-de-tucano, coca-del-campo.

Fenologia: Floresce e frutifica quase ao mesmo tempo, florescendo de fevereiro até dezembro e frutificando de novembro a março. São frequientes plantas com botões, flores e frutos.

Distribuição: Rio Grande do Sul, Santa Catarina, Paraná, São Paulo, Rio de Janeiro, Minas Gerais, Goiás, Mato Grosso, Argentina, Paraguai e Bolívia. Espécie xilopodífera ocorrendo tanto no cerrado, mais propriamente nas orlas, quanto nos campos rochosos e em margens de rios, entretanto, em locais secos. As flores são bastante grandes em relação às folhas.

Usos: seus frutos são procurados pelas aves (Amaral Júnior, 1980).

Material examinado: PARANÁ: Almirante Tamandaré, Rio Barigüi, II.1982, G. Hatschbach et al. 44604 (MBM). Balsa Nova, Campina da Cascavel, XI.1976, G. Hatschbach 38181 (MBM). Bocaiúva do Sul, XII.1978, G. Hatschbach 41917 (MBM). Campo Largo, XII.1949, G. Hatschbach 1633 (PACA). Castro, P. F. de Caxambu, .1984, J. Mattos et al. 28762 (HAS). Jaguariaíva, Faz. Chapada Sto.
Antônio, VI.1994, J.O. Mendonça \& A. Uhlmann 12 (UPCB). Lapa, Rio Passa Dois, II.1981, G. Hatschbach 44401 (MBM). Palmeira, Rio dos Papagaios, VI.1994, J.O. Mendonça 17 \& A.C. Cervi (UPCB). Ponta Grossa, Vila Velha, XI.1989, J. Mattos et al. 26789 (HAS). Rio Branco do Sul, II.1982, G. Hatschbach 44549 (MBM). São José dos Pinhais, Rio Pequeno, III.1980, G. Hatschbach 42767 (MBM).

IV - Seção Macrocalyx O. E. Schulz 15. Erythroxylum suberosum A. St.-Hil. in Pl. Usuell. Bras. 69, fig. a. 1824, Fl. Bras. Mer. II: 95. 1829; Mart. in Abhandl. Akad. München III. 2: 376. 1843; Peyr. in Mart. Fl. Bras. XII. 1: 170. 1878; O.E. Schulz in Engler Das Pflanzenr. IV. 137. 29: 27. 1907.

Nome vulgar: galinha-choca, mercúrio-docampo, sessenta-e-dois, azougue-do-campo, cabelo-de-negro.

Fenologia: Floresce de agosto a dezembro, frutifica de setembro a janeiro.

Distribuição: Paraná, São Paulo, Rio de Janeiro, Espírito Santo, Minas Gerais, Mato Grosso do Sul, Mato Grosso, Goiás, Distrito Federal, Bahia, Alagoas, Pernambuco, Ceará, Maranhão, Amapá, Amazonas, Acre, Paraguai, Bolívia e Venezuela.

Usos: Fornece madeira vermelho-escura, apesar de Corrêa (1984), citar que a madeira desta espécie é própria para marcenaria e carpintaria, isto não é possível, pois a espessura e rigidez do caule não permitem tal uso. A casca suberosa é adstringente útil para curtume, também fornece matéria tintorial castanho-avermelhada, que dizem ter fixidez. Os frutos são dados às galinhas, cujo choco pretende-se evitar.

Material examinado: PARANÁ: Arapoti, Chapada Santo Antônio, X.1968, G. Hatschbach 19973 (MBM). Campo Mourão, XII.1965, G. Hatschbach et al. 13263 (MBM). Jaguariaíva, XI.1993, J.O. Mendonça \& A.C. Cervi 07 (UPCB). Piraí do Sul, Faz. das Almas, IV.1987, Y.S. Kuniyoshi \& C.V. Roderjan 5177 (EFC). Rio Branco do Sul, Serra do Caete, X.1979, G. 
Hatschbach 42516 (MBM). Sengés, Res. Ecol. Pisa Florestal, III.1995, J. O. Mendonça \& Uhlmann 39 (UPCB). Tibagi, Canyon Guartelá, XII.1993, J.O. Mendonça A.C. Cervi 09 (UPCB).

Comentários: Espécie típica do campo cerrado; após as queimadas rebrota vigorosamente.

\section{Considerações sobre a nomenclatura do gênero}

O nome correto para o gênero, vem sendo, há muito discutido. Alguns autores como, A. SaintHilaire (1824, 1829), Peyritsch (1878), O.E. Schulz (1907), Plowman (1976) e Amaral Júnior (1980), adotam o nome Erythroxylum P. Browne (Browne, 1756). Outros como, Martius (1843) e Machado (1970, 1972), optam por Erythroxylon L. (Linnaeus, 1759). Há muitos argumentos favoráveis e contrários para ambos os casos e, acreditamos ser necessário um aprofundamento nessa questão. Nossa posição, é mais conservadora em favor de Erythroxylum P. Browne.

\section{CONCLUSÕES}

$\mathrm{O}$ gênero Erythroxylum P. Browne está representado no Estado do Paraná por 15 espécies: E. ambiguum, E. amplifolium, $E$. anguifugum, $E$. argentinum, $E$. buxus, $E$. campestre, E. cuneifolium, E. cuspidifolium, E. deciduum, E. gonocladum, E. microphyllum, E. myrsinites, E. nanum, E. pelleterianum e E. suberosum.

Um sinônimo novo é proposto: $E$. cuneifolium (Mart.) O.E. Schulz var. squarrosum O.E. Schulz, como sinônimo de E. cuneifolium (Mart.) O.E. Schulz.

Quanto à aplicação correta do nome do gênero, acreditamos ser necessário um aprofundamento maior no estudo dessa questão.

Todas as argumentações favoráveis e contrárias à manutenção do nome existente, deveriam ser encaminhadas ao Comitê Permanente de Nomenclatura da Associação Internacional de
Taxonomia Vegetal (IAPT), para apreciação e parecer final.

\section{AGRADECIMENTOS}

Os autores agradecem ao Curso de PósGraduação em Botânica da Universidade Federal do Paraná, onde desenvolveu-se este trabalho, à CAPES pelo apoio financeiro, ao Prof. Dr. Ayrton Amaral Jr., do Departamento de Botânica da UNESP-Botucatu, pela revisão e sugestões e ao $\mathrm{Eng}^{\circ}$.Agr ${ }^{\circ}$. M.Sc. Cláudio J.F. Alves de Brito na preparação do manuscrito.

\section{REFERÊNCIAS BIBLIOGRÁFICAS}

Amaral Jr., A. (1973), O gênero Erythroxylum no município de Botucatu, São Paulo. Botucatu, Tese (Doutorado em Ciências) Faculdade de Ciências Médicas e Biológicas de Botucatu, UNESP.

Amaral Jr., A. Eritroxiláceas. (1980), Flora Ilustrada Catarinense, Itajaí, 64p.

Amaral Jr., A. \& Mendonça, J.O. (1998), Erythroxylum perpusillum Fern. Casas, um novo sinônimo em Erythroxylaceae. Pabstia, $9(1), 1-8$.

Browne, P. (1756), The Civil and Natural History of Jamaica. London: T. Osborne and J. Shipton.

Corrêa, M.P. (1984), Dicionário de plantas úteis do Brasil. Rio de Janeiro: Imprensa Nacional, $6 \mathrm{v}$.

Cronquist, A. (1981), An integrated system of classification of flowering plants. New York: Columbia University Press, $1262 \mathrm{p}$.

Holmgren, P.; Keuken, W.; Schfield, E. (1990), Index Herbariorum - Part I - the herbaria of the world. New York: New York Botanical Garden, 693 p.

Linnaeus, C. (1759), Erythroxylon. Systema Naturae, v. 2, 10 ed. Estocolmo, 1035.

Machado, E.C. (1970), Nomenclatura del gênero Erythroxylon L. Anales Científicas (La Molina), 8 (1-2), 17-18.

Machado, E.C. (1972), El genero Erythroxylon en el Peru. Raymondiana, 5, 5-101.

Martius, K.F.P. (1843), Beiträge zur kenntnis der Gattung Erythroxylon. Abhandl. Akad. 
München III, 280-410.

Mendonça, J.O. (1996), O gênero Erythroxylon Linnaeus (Erythroxylaceae) no Estado do Paraná, Brasil. Tese (Mestrado em Botânica). Universidade Federal do Paraná, Setor de Ciências Biológicas, Curitiba.

Occhioni, P.; Hatschbach, G. (1972), A vegetação dos ervais do Paraná - I. Leandra, 2 (3), 5-59.

Peyritsch, J. (1878), Erythroxylaceae. In: MARTIUS. Flora Brasiliensis, 12 (1), 126180.

Plowman, T. (1976), Orthography of Erythroxylum (Erythroxylaceae). Taxon, 25 (1), 141-144.
PLOWMAN, T. (1984), New taxa of Erythroxylum (Erythroxylaceae) from the Amazon Basin. Acta Amazonica, 14 (1-2), 117-143.

Adford, D. A. E. ; Dickson, W. C. et al. (1974), Vascular plant systematic. New York: Hoyer \& Row, 1974. $891 \mathrm{p}$.

Saint-Hilaire, A.F.C.P. (1824), Plants Usuelles des Brasiliens. Paris: Grimbert Libraire.

Saint-Hilaire, A.F.C.P. (1829), Erythroxylaceae. In: Flora Brasiliae Meridionalis II, pp. 6672.

Schulz, O.E. (1907), Erythroxylaceae. In Engler, A. Das Pflanzenreich: regni vegetabilis conspectus. Weinheim: Verlag von H. R. Engelmann, 29 (134), 1-76.

Sobral, M. (1987), Erythroxylum (Erythroxylaceae) no Rio Grande do Sul. Pesquisas Botânica, 38, 7-42. 